\title{
The Cosmic Neutrino Background and the age of the Universe
}

\author{
Francesco de Bernardis ${ }^{1}$, Alessandro Melchiorri ${ }^{1}$, Licia $\operatorname{Verde}^{2,3}$ \\ and Raul Jimenez ${ }^{2,3}$ \\ ${ }^{1}$ Physics Department and Sezione INFN, University of Rome \\ "La Sapienza", P.le Aldo Moro 2, 00185 Rome, Italy \\ ${ }^{2}$ ICREA \& Institute of Space Sciences (CSIC-IEEC), Campus \\ UAB, Bellaterra, Spain. \\ ${ }^{3}$ Dept. of Astrophysical Sciences, Princeton University, Ivy \\ lane, Princeton, NJ 08544, USA.
}

\begin{abstract}
.
We discuss the cosmological degeneracy between the age of the Universe, the Hubble parameter and the effective number of relativistic particles $N_{\text {eff }}$. We show that independent determinations of the Hubble parameter $H(z)$ such as those recently provided by [1, combined with other cosmological data sets can provide the most stringent constraints on $N_{\text {eff }}$, yielding $N_{\text {eff }}=3.7_{-1.2}^{+1.1}$ at $95 \%$ confidence level. A neutrino background is detected with high significance: $N_{\text {eff }}>1.8$ at better than $99 \%$ confidence level. Constraints on the age of the Universe in the framework of an extra background of relativistic particles are improved by a factor 3 .
\end{abstract}

Keywords: Neutrinos: cosmological neutrinos, neutrino properties- 


\section{Introduction}

The recent measurements of cosmic microwave background (CMB) anisotropies and polarization [2, 3], alone or in combination with other cosmological data sets, have provided confirmation of the standard cosmological model and an accurate determation of some of its key parameters. In particular, the new determination of the age of the Universe $13.8 \pm 0.3$ Gyrs improves by an order of magnitude previous determinations from, e.g., cosmochronology of long-lived radioactive nuclei [4] and population synthesis of the oldest stellar populations [5, 6, 7].

It is important however to constrain possible deviations from the standard cosmological model. Here we concentrate on constraints on the amount of relativistic energy density at recombination: cosmological results can be dramatically affected if assumptions about the physical energy density in relativistic particles $\omega_{\text {rel }}$ are relaxed. The shape of the CMB angular power spectrum is sensitive to the epoch of matterradiation equality: a change in $\omega_{\text {rel }}$ can be compensated by a change in the physical cold dark matter density $\omega_{c}$, in the Hubble constant $H_{0}$ and, to less extent, in the power spectrum spectral slope $n_{s}$ [8]. Small scales observables (such as the CMB damping tail and large-scale structure) are also affected e.g., [9, 10, 11, 16].

In the standard model, $\omega_{\text {rel }}$ includes photons and neutrinos, and is often parameterized in terms of the equivalent number of standard massless neutrinos species $N_{\text {eff }}$; in particular, $\omega_{\text {rel }}=\omega_{\gamma}+N_{\text {eff }} \omega_{\nu}$, where $\omega_{\gamma}$ is the energy density in photons and $\omega_{\nu}$ is the energy density in one active neutrino. Measuring $\omega_{\text {rel }}$ thus gives a direct observation on the effective number of neutrinos, $N_{\text {eff }}$. The standard model predicts three neutrino species; corrections to account for QED effects and for neutrinos being not completely decoupled during electron-positron annihilation imply $N_{\mathrm{eff}}^{S M}=3.04$ [17, 18, 19, 20, 21]. Any light particle that does not couple to electrons, ions and photons will act as an additional relativistic species.

Departures from the standard model which are described by a deviation $N_{\text {eff }} \neq 3$ can arise from the decay of dark matter particles [22, 23, 24, 25], quintessence [26], exotic models [27], and additional hypothetical relativistic particles such as a light majoron or a sterile neutrino. Such hypothetical particles are strongly constrained from standard big bang nucleosynthesis (BBN), where the allowed extra relativistic degrees of freedom

are $N_{\mathrm{eff}}^{\mathrm{BBN}}=3.1_{+1.4}^{-1.2}$ (see e.g. [28]). When comparing $N_{\text {eff }}$ constraints from BBN and $\mathrm{CMB}$, one should keep in mind that they rely on different physics and the energy density in relativistic species may easily change from the time of $\mathrm{BBN}(T \sim \mathrm{MeV})$ to the time of last rescattering $(T \sim \mathrm{eV})$ in several non-standard models. Moreover, the two estimates are affected by different systematics (see [28]).

Cosmological data analyses with variations in $N_{\text {eff }}$ have been recently undertaken by many authors $[29,14,11,15,12,13,30,31,32,10,28,[52]$. Here, we first analyze the degeneracy of cosmological parameters and in particular the age of the Universe, with $N_{\text {eff }}$, and then show how determinations of the Hubble parameter can help break this degeneracy. We show that the recent determinations of $H_{0}$ from the HST key project 
[33] and $H(z)$ provided by [1] (SVJ; based on [34] and references therein) can provide, when combined with CMB and other cosmological data, new bounds on $N_{\text {eff }}$.

\section{Data Analysis: Method and Results}

The method we adopt is based on the publicly available Markov Chain Monte Carlo package cosmomc [35]. We start by considering a standard LCDM model described by the following set of cosmological parameters, adopting flat priors on them: the physical baryon and CDM densities, $\omega_{b}=\Omega_{b} h^{2}$ and $\omega_{c}=\Omega_{c} h^{2}$, the ratio of the sound horizon to the angular diameter distance at decoupling, $\theta_{s}$, the scalar spectral index, $n_{s}$ and amplitude $A_{s}$, and the optical depth to reionization, $\tau$. For all these parameters the chosen boundaries of the priors do not affect the cosmological constraints. To these parameters we add the possibility of having an extra-background of relativistic particles (parametrized by $N_{\text {eff }} \neq 3.04$, see e.g. [8], with a flat prior $\left.0 \leq N_{\text {eff }} \leq 15\right) \ddagger$. Later on, when we consider the possibility of a dark energy equation of state $w \neq-1$, we assume a flat prior on $w$. Temperature, cross polarization and polarization CMB fluctuations from the WMAP 3 year data [2, 3, 36, 37] are considered. WMAP data are combined with the following data sets: higher resolution CMB experiments BOOMERanG, ACBAR, CBI and VSA [38, 39, 40, 41, 42, 43, 44, 45, 46], the power spectrum of galaxies from the Sloan Digital Sky Survey (SDSS) [47] and Two degree field Galaxy Redshift Survey (2dFGRS) [48] on linear scales $\left(k<0.2(\mathrm{Mpc} / \mathrm{h})^{-1}\right)$; the galaxy bias $b$ is considered as an additional nuisance parameter and is marginalized over. Constraints obtained from the supernova (SN-Ia) luminosity measurements are also included with data set from [50, 49]. This combination is referred to as "WMAP+ALL".

Finally, we consider Hubble key project determination of the Hubble constant [33] (HST) and the SVJ [1] determination of the redshift dependence of the Hubble parameter $H(z)$ from observations of passively evolving galaxies (SVJ).

Since the SDSS and 2dFGRS data differ in the shapes of the two measured power spectra, leading to a disagreement in their best fit values for $N_{\text {eff }}$ [2], we also consider the reduced combination of WMAP alone and WMAP+SDSS (this is the combination of CMB and large scale structure which give the weakest constraints in $N_{\text {eff }}$ ). As we will show below, this reduced combination with the addition of the Hubble parameter determinations provide constraints virtually indistinguishable form those obtained with the "ALL" data set.

When $\omega_{\text {rel }}$ (or $N_{\text {eff }}$ ) is considered as a free parameter the constraint on the age of the Universe , $t_{0}$, from cosmological data changes from $13.84 \pm 0.28$ Gyrs to $13.8_{-3.2}^{+2.3}$ Gyrs: there is a strong degeneracy between $\omega_{\text {rel }}$ and $\omega_{m}$ which is mostly driven by a

\$ While it is common to assume a flat prior on $\theta_{s}$, the standard version of cosmomc uses a fitting formula to convert between $\theta_{s}$ and $H$ which is valid for $N_{r m e f f} \equiv 3.04$. Thus running the standard cosmomc with a flat prior on $\theta_{s}$ and $N_{\text {eff }} \neq 3.04$ is equivalent to using a distorted prior on $H$. We find that this does not affect significantly the cosmological constraints, in particular for $50<H_{0}<80$ the difference is completely negligible. 
The Cosmic Neutrino Background and the age of the Universe

Table 1. Cosmological constraints (95\% c.l.) on the number of relativistic particles and other cosmological parameters from WMAP alone, WMAP+ALL, adding HST and SVJ data sets (see text for more details; a conservative prior $H_{0} \leq 100 \mathrm{Km} / \mathrm{s} / \mathrm{Mpc}$ is included in the WMAP alone case)

\begin{tabular}{|l||c|c|c|c|}
\hline Parameter & $\begin{array}{c}\text { WMAP alone } \\
N_{\text {eff }}=3.04\end{array}$ & WMAP alone & $\begin{array}{c}\text { WMAP+ALL } \\
N_{\text {eff }}=3.04\end{array}$ & WMAP+ALL \\
\hline$N_{\text {eff }}$ & - & $5.5_{-3.9}^{+4.0}$ & - & $3.3_{-2.3}^{+4.0}$ \\
$H_{0}$ & $73.2_{-3.2}^{+3.1}$ & $82_{-16}^{+18}$ & $70.8_{-3.1}^{+3.3}$ & $71_{-12}^{+21}$ \\
$\omega_{c}$ & $0.1054_{-0.0077}^{+0.0078}$ & $0.15_{-0.07}^{+0.08}$ & $0.1087_{-0.0078}^{+0.0088}$ & $0.113_{-0.037}^{+0.086}$ \\
$\Omega_{m}$ & $0.241_{-0.034}^{+0.034}$ & $0.25_{-0.06}^{+0.07}$ & $0.262_{-0.032}^{+0.036}$ & $0.265_{-0.039}^{+0.038}$ \\
age(Gyr) & $13.73_{-0.30}^{+0.31}$ & $12.4_{-6.0}^{+5.5}$ & $13.84_{-0.28}^{+0.28}$ & $13.8_{-3.2}^{+2.3}$ \\
\hline & WMAP+ALL & WMAP+ALL & WMAP+ALL & WMAP alone \\
Parameter & $+\mathrm{HST}$ & $+\mathrm{SVJ}$ & $+\mathrm{HST}+\mathrm{SVJ}$ & $+\mathrm{SVJ}$ \\
\hline$N_{\text {eff }}$ & $3.03_{-1.7}^{+2.2}$ & $3.8_{-1.15}^{+1.2}$ & $3.71_{-1.08}^{+1.17}$ & $4.0_{-1.2}^{+1.2}$ \\
$H_{0}$ & $70.5_{-9.5}^{+10.5}$ & $73.8_{-6.5}^{+6.6}$ & $73.5_{-5.9}^{+6.1}$ & $75.5_{-7.4}^{+8.1}$ \\
$\omega_{c}$ & $0.109_{0.029}^{+0.045}$ & $0.121_{-0.021}^{+0.024}$ & $0.120_{-0.020}^{+0.023}$ & $0.124_{-0.027}^{+0.035}$ \\
$\Omega_{m}$ & $0.264_{-0.036}^{+0.035}$ & $0.262_{-0.040}^{+0.36}$ & $0.263_{-0.036}^{+0.035}$ & $0.258_{-0.058}^{+0.045}$ \\
age(Gyr) & $13.9_{-2.0}^{+1.8}$ & $13.27_{-0.97}^{+1.1}$ & $13.31_{-0.91}^{+1.0}$ & $13.1_{-1.1}^{+1.1}$ \\
\hline
\end{tabular}

Table 2. As table 2 but for WMAP+SDSS. (see text for more details).

\begin{tabular}{|l||c|c|c|c|c|}
\hline & $\begin{array}{c}\text { WMAP+ } \\
\text { SDSS } \\
N_{\text {eff }}=3.04\end{array}$ & $\begin{array}{c}\text { WMAP+ } \\
\text { SDSS }\end{array}$ & $\begin{array}{c}\text { WMAP+ } \\
\text { SDSS } \\
+ \text { HST }\end{array}$ & $\begin{array}{c}\text { WMAP+ } \\
\text { SDSS } \\
+ \text { SVJ }\end{array}$ & $\begin{array}{c}\text { WMAP+ } \\
\text { SDSS } \\
+ \text { HST+SVJ }\end{array}$ \\
\hline$N_{\text {eff }}$ & - & $7.1_{-5.1}^{+7.1}$ & $4.3_{-1.6}^{+2.8}$ & $4.0_{-1.1}^{+1.2}$ & $4.0_{-1.1}^{+1.1}$ \\
$H_{0}$ & $70.9_{-4.7}^{+5.3}$ & $86_{-21}^{+24}$ & $75_{-21}^{+13}$ & $74.5_{-7.3}^{+7.7}$ & $74.0_{-6.6}^{+6.9}$ \\
$\omega_{c}$ & $0.110_{-0.013}^{+0.013}$ & $0.18_{-0.086}^{+0.13}$ & $0.132_{-0.042}^{+0.068}$ & $0.128_{-0.024}^{+0.027}$ & $0.127_{-0.024}^{+0.027}$ \\
$\Omega_{m}$ & $0.265_{-0.056}^{+0.062}$ & $0.265_{-0.065}^{+0.077}$ & $0.270_{-0.060}^{+0.069}$ & $0.271_{-0.057}^{+0.065}$ & $0.274_{-0.065}^{+0.062}$ \\
age & $13.77_{-0.30}^{+0.29}$ & $11.5_{-2.7}^{+3.3}$ & $13.0_{-2.0}^{+2.0}$ & $13.0_{-0.92}^{+1.0}$ & $13.1_{-0.87}^{+0.96}$ \\
\hline
\end{tabular}

degeneracy between $N_{\text {eff }}$ and $H_{0}$. This is shown in the first four columns of table 1 (for WMAP dataset alone and WMAP+ALL), and the first two columns of table 3 (for WMAP+SDSS).

In the first two columns of Table I we show the constraints from WMAP alone: including variations in $N_{\text {eff }}$ strongly affects the determination of most of the parameters as the Hubble constant and the matter density. In the last column of Table I, where we report the constraints obtained from WMAP alone + the SVJ dataset. The constraints on the age of the Universe are improved by a factor 3 while constraints on the Hubble parameter are now a factor 2 better. Age and $H(z)$ data are therefore extremely useful in breaking the degeneracy. Consequently the WMAP+SVJ analysis yields $N_{\text {eff }}=4.0_{-1.2}^{+1.2}$. 


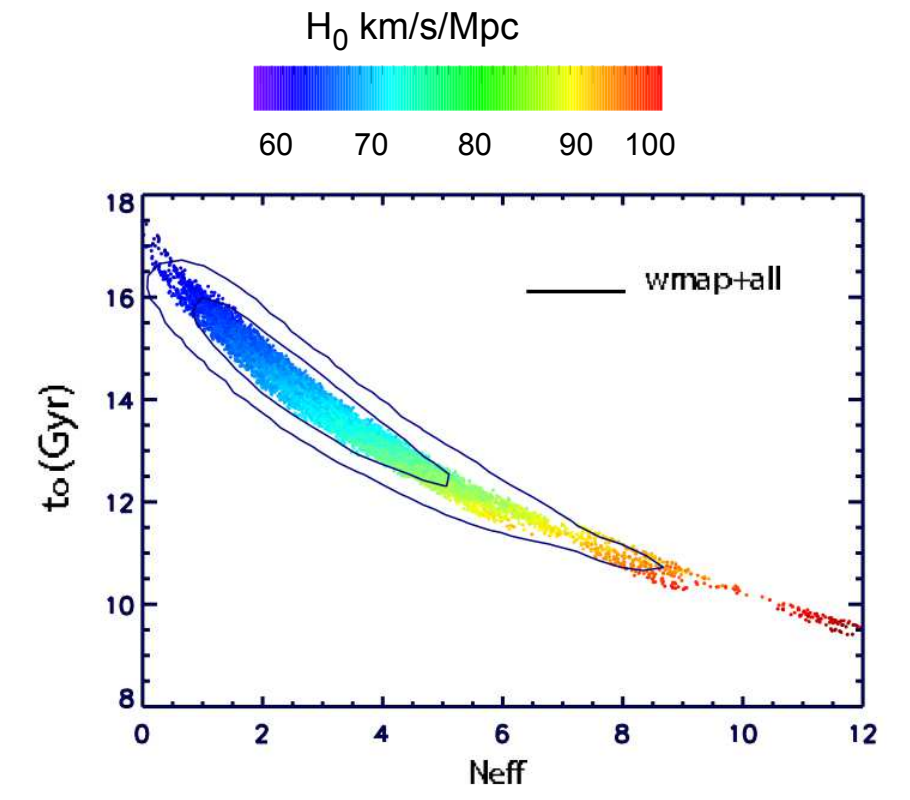

Figure 1. Constraints on the $t_{0}-N_{\text {eff }}$ plane from current cosmological data (WMAP+ALL). A degeneracy between the two parameters (and the Hubble constant) is evident. An extra background of relativistic particles with respect to the standard model prediction of $N_{\text {eff }}=3.04$ is in agreement with current cosmological data for ages of the Universe $t_{0}<13 \mathrm{Gyr}$, and Hubble constant $H_{0}>70 \mathrm{~km} / \mathrm{s} / \mathrm{Mpc}$.

It is useful to examine the constraints from the cosmological dataset combination WMAP + ALL on the $N_{\text {eff }}-t_{0}$ plane as shown in Fig. 1. A strong degeneracy is present and the relativistic background is poorly constrained: values of $N_{\text {eff }}$ as large as 8 are allowed at $95 \%$ c.l. and letting $N_{\text {eff }}$ vary results in much weaker constraints on the current age of the Universe $t_{0}$. As we can see, even if one includes most of the current cosmological datasets, except $H(z), N_{\text {eff }}$ and $t_{0}$ are poorly constrained.

Figure 2 shows how $H(z)$ constraints reduce the parameter space allowed by the rest of the data combination while table 2 (WMAP+ALL) and 3 (WMAP+SDSS) show how the degeneracy can be broken and constraints on $N_{\text {eff }}$ can be improved by adding Hubble constant constraints; here HST denotes the HST Hubble key project $H_{0}$ determination [33] and SVJ denotes [1]. The constraints obtained from the combination $\mathrm{WMAP}+\mathrm{ALL}+\mathrm{HST}+\mathrm{SVJ}$ are virtually indistinguishable from those obtained from the combination WMAP+SDSS+HST+SVJ. This implies that the tension between 2dFGRS and SDSS does not affect the result and that the combination high-resolution CMB experiments+ 2dFGRS+Supernovae (SNLS \& GOLD) does not add a significant amount of information once the $H$ determinations are included.

We summarize the constraints on $N_{\text {eff }}$ in figure 3, where we show the likelihood $(L)$ as a function of $N_{\text {eff }}$ marginalized over all other parameters, for the case WMAP+ALL (top) and WMAP+SDSS (bottom). Lines corresponds to the data set combination without $H$ determinations (dotted), adding HST (dashed) and adding both HST and SVJ (solid). When the Hubble parameter determinations are added a neutrino background is detected at high significance: $N_{\text {eff }}>1.8$ at better than $99 \%$ confidence 


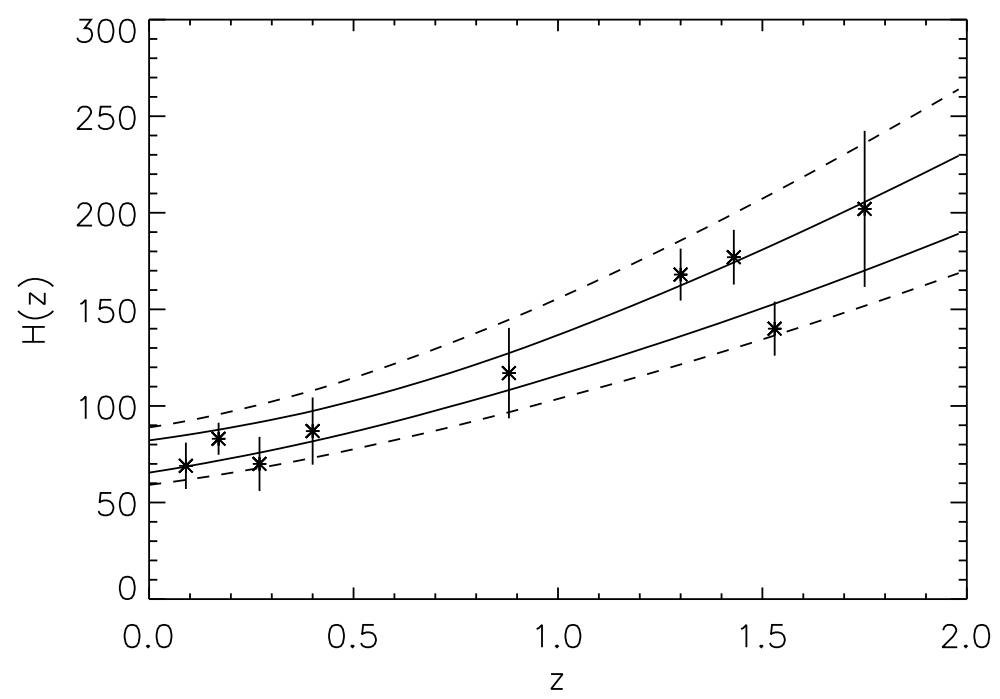

Figure 2. The dashed lines show the range of expansion histories, $H(z)$, allowed at the $68 \%$ c.l. by the data set WMAP+SDSS +HST. The points with error-bars are the $H(z)$ determinations from [1](SVJ) and the solid line shows the range of expansion histories allowed at the $68 \%$ c.l. after adding the constraints from these data.

level.

Table 3. Cosmological constraints (95\% c.l.) on the number of relativistic particles allowing for a dark energy equation of state $w \neq-1$ from WMAP $+2 \mathrm{dF}+\mathrm{SN}-\mathrm{Ia}$ and when adding the SVJ data.

\begin{tabular}{|l||c|c|}
\hline Parameter & $\begin{array}{c}\text { WMAP+2dF } \\
+ \text { SN-Ia }\end{array}$ & $\begin{array}{c}\text { WMAP+2dF } \\
+ \text { SN-Ia+SVJ }\end{array}$ \\
\hline$N_{\text {eff }}$ & $3.5_{-3.5}^{+3.8}$ & $3.8_{-1.1}^{+1.1}$ \\
$w$ & $-0.96_{-0.15}^{+0.14}$ & $-0.95_{-0.16}^{+0.14}$ \\
$H_{0}$ & $72.7_{+13.7}^{-13.2}$ & $74.2_{-7.2}^{+6.8}$ \\
$\omega_{c}$ & $0.11_{-0.04}^{+0.07}$ & $0.116_{-0.023}^{+0.027}$ \\
$\Omega_{m}$ & $0.25_{-0.04}^{+0.04}$ & $0.251_{-0.039}^{+0.042}$ \\
age(Gyr) & $13.7_{-2.9}^{+2.5}$ & $13.2_{-1.0}^{+1.1}$ \\
\hline
\end{tabular}

Finally, we consider the possibility of a dark energy equation of state $w \neq-1$. We include WMAP $+2 \mathrm{dF}$ and SN-Ia datasets and we report the constraints in table III. The table shows that allowing $w \neq-1$ produces little effect on $N_{\text {eff }}$ when the SVJ data are included.

Very recently, several works have bounded the cosmological neutrino background using cosmological data: [51] and [28] report $N_{\text {eff }}=4.6_{-1.6}^{+1.5}$. This limit, which is in excellent agreement with the limit presented here, is obtained by including Lyman$\alpha$ and baryonic acoustic oscillation data, which are affected by completely different 

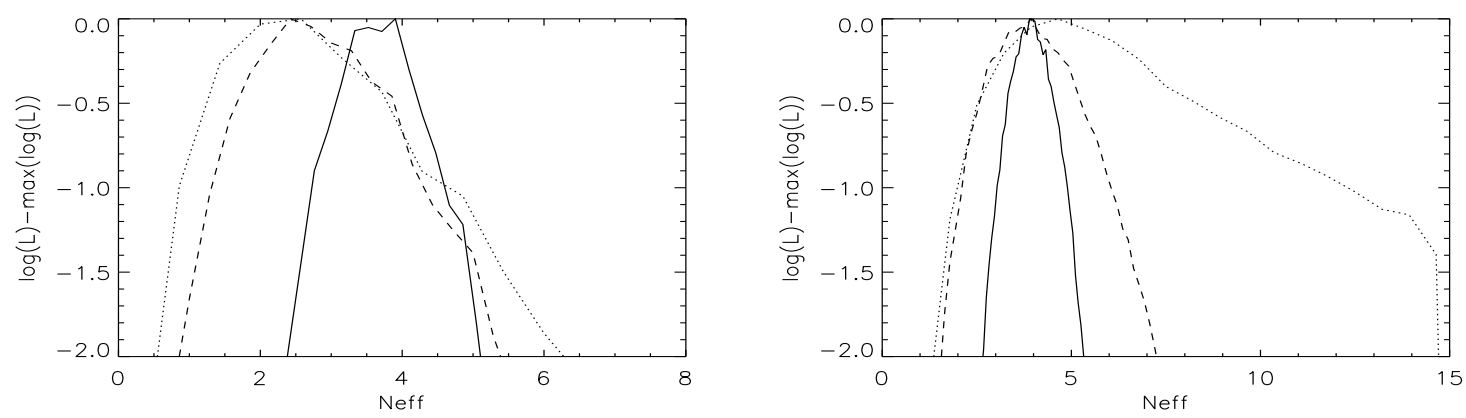

Figure 3. Logarithm of the Likelihood $(\ln (L))$ as a function of $N_{\text {eff }}$ marginalized over all other parameters and normalized to be 0 at the maximum. Top: WMAP+ALL. Bottom: WMAP+SDSS. Lines corresponds to the data set combination without $H$ determinations (dotted), adding HST (dashed), adding HST and SVJ (solid).

systematics from the Hubble parameter determinations used here (see [52] for a discussion; systematics in the SVJ method are described and quantified in [34, 54]).

WMAP +SVJ (see Table I) combination which includes information from 2 datasets produces $40 \%$ more stringent constraints than those reported in these analyses which includes more than 5 datasets.

\section{Conclusions}

We have considered how the Hubble parameter determinations of $H_{0}$ from the HST key project [33] and of $H(z)$ from passively evolving galaxies [1] (SVJ) improve constraints on the physical energy density in relativistic particles, parameterized as an effective number of massless neutrino species $N_{\text {eff }}$ which can deviate from the standard model prediction of $N_{\text {eff }}^{S M}=3.04$. We find $N_{\text {eff }}=3.7_{-1.1}^{+1.2}(95 \%$ c.l. $)$ from the dataset combination WMAP + ALL $+\mathrm{HST}+\mathrm{SVJ}$ and $N_{\text {eff }}=4.0_{-1.1}^{+1.1}(95 \%$ c.l. $)$ from the reduced combination WMAP+SDSS+HST+SVJ. The WMAP+SDSS combination was chosen over e.g. WMAP + other galaxy surveys, as SDSS is the large-scale structure data set yielding the weakest constraints on $N_{\text {eff }}$ when combined with WMAP. These constraints are in good agreement with the standard model prediction. The addition of Hubble parameter determinations to the other cosmological data-sets break the degeneracy between $N_{\text {eff }}$ and the age of the Universe. The nature of this degeneracy implies that there is not a range of redshift where $H(z)$ determinations would yield the best constraints on $N_{\text {eff }}$. On the other hand, for a redshift range $0.8<z<2.0$ the corresponding age of the Universe is about 6.5 to 3 Gyr. At these ages and for solar metallicity, the UV spectra of galaxies $(2300<\lambda / \AA<4000)$ are dominated by main sequence stars of $\mathrm{F}$ and $\mathrm{G}$ class similar to our Sun [53]. This is a stage of stellar evolution that can be modeled accurately unlike stages in the post main-sequence dominated by mass-loss events. This will therefore be the preferred redshift range to obtain further observations of passively evolving high-redshift galaxies to further constrain the number 
of relativistic species.

Future baryon acoustic oscillations spectroscopic surveys will yield very accurate measurements of $H(z)$, which can be used to further constrain $N_{\text {eff }}$. Forecasts for the $N_{\text {eff }}$ constraints from these surveys in combination with future CMB data, will be presented elsewhere.

\section{Acknowledgments}

We acknowledge the use of the Legacy Archive for Microwave Background Data Analysis (LAMBDA). Support for LAMBDA is provided by the NASA Office of Space Science.

LV and RJ thank D. Spergel for discussions. We thank N. Wright and S. Bashinsky for carefully reading the manuscript and for comments.

\section{References}

[1] J. Simon, L. Verde and R. Jimenez, Phys. Rev. D 71 (2005) 123001

[2] D. N. Spergel et al., ApJS, 170 (2007) 37

[3] D. N. Spergel et al., ApJS, 148 (2003) 175

[4] Frebel, A., Christlieb, N., Norris, J. E., Thom, C., Beers, T. C., \& Rhee, J. 2007, Astrophys. J. Lett., 660, L117

[5] Jimenez, R., Thejll, P., Jorgensen, U. G., MacDonald, J., \& Pagel, B., MNRAS, 282 (1996) 926;

[6] Dunlop, J., Peacock, J., Spinrad, H., Dey, A., Jimenez, R., Stern, D., \& Windhorst, R., Nature, 381 (1996) 581

[7] Krauss, L. M., \& Chaboyer, B., Science, 299 (2003) 65

[8] R. Bowen et al., Mon. Not. Roy. Astron. Soc. 334 (2002) 760

[9] Hall, L.J. and Olivier, S., 2004, Nuclear Physics B Proceedings Supplements, 137, 269

[10] Hannestad, S. , Journal of Cosmology and Astro-Particle Physics, 2 (2005) 11

[11] E. Pierpaoli, Mon. Not. Roy. Astron. Soc., 342 (2003) L63

[12] K. Ichikawa, M. Kawasaki and F. Takahashi, JCAP 0705, 007 (2007)

[13] K. Ichikawa, M. Kawasaki, K. Nakayama, M. Senami and F. Takahashi, JCAP 0705, 008 (2007)

[14] S. H. Hansen, G. Mangano, A. Melchiorri, G. Miele and O. Pisanti, Phys. Rev. D 65 (2002) 023511

[15] Peacock, J. A., astro-ph/0309238

[16] Bashinsky, S. \& Seljak, U. 2004, Phys. Rev. D, 69, 083002, Bashinsky, S. 2007, arXiv:0707.0692,

[17] Dolgov, A. D., Hansen, S. H., \& Semikoz, D. V. 1999, Nucl. Phys. B543, 269

[18] Gnedin, N. Y. \& Gnedin, O. Y. 1998, ApJ, 509, 11

[19] Lopez R.E., Dodelson S., Heckler A., Turner M.S., 1999, Phys. Rev. Lett. 82, 3952

[20] Mangano G., Miele G., Pastor S., Peloso M., 2002, Phys.Lett. B534, 8;

[21] Mangano, G., Miele, G., Pastor, S., Pinto, T, Pisanti, O. \& Serpico, P. D. 2005, Nucl. Phys. B729, 221

[22] Bonometto S., Pierpaoli E., 1998, New Astronomy, 3 , 391

[23] Lopez R.E.,Dodelson S., Sherrer R.J., Turner M.S., 1998, Phys. Rev. Lett. 81, 3075

[24] Hannestad S., 1998, Phys.Rev.Lett. 80, 4621

[25] Kaplinghat M., Turner M.S., 2001, Phys. Rev. Lett. 86, 385

[26] Bean R., Hansen S.H., Melchiorri A., 2001, Phys. Rev. D 64, 103508

[27] H. Davoudiasl, arXiv:0705.3636

[28] G. Mangano, A. Melchiorri, O. Mena, G. Miele and A. Slosar, JCAP 0703 (2007) 006

[29] Hannestad, S., Journal of Cosmology and Astro-Particle Physics, 5 (2003) 4 
[30] Crotty, P., Lesgourgues, J., \& Pastor, S. (2003) PRD, 67, 123005

[31] Elgarøy, Ø., \& Lahav, O. (2003), Journal of Cosmology and Astro-Particle Physics, 4, 4

[32] Barger, V., Kneller, J. P., Langacker, P., Marfatia, D., \& Steigman, G. 2003, Physics Letters B, 569,123

[33] Freedman, W. L., et al. 2001, ApJ, 553, 47

[34] R. Jimenez, L. Verde, T. Treu, and D. Stern, ApJ 593, 622 (2003).

[35] A. Lewis and S. Bridle, Phys. Rev. D 66 (2002) 103511

[36] L. Page et al., ApJS, 170, 335, 2007

[37] G. Hinshaw et al., ApJS, 170, 288, 2007

[38] Ruhl, J. E., et al. 2003, ApJ, 599, 786;

[39] Montroy, T. E., et al. 2005, ApJ;

[40] Piacentini, F., et al. 2005,ArXiv Astrophysics e-prints;

[41] Kuo, C. L., et al. 2004, ApJ, 600, 32;

[42] Mason, B. S., et al. 2003, ApJ, 591, 540;

[43] Sievers, J. L., et al. 2003, ApJ, 591, 599;

[44] Pearson, T. J., et al. 2003, ApJ, 591, 556;

[45] Readhead, A. C. S., et al. 2004a, ApJ, 609, 498;

[46] Dickinson, C., et al. 2004, MNRAS, 353, 732

[47] M. Tegmark et al., Astrophys. J. 606, 702 (2004).

[48] S. Cole et al., MNRAS 362, 505 (2005).

[49] P. Astier et al., Astron. Astrophys. 447, 31 (2006).

[50] A.G. Riess et al., Astrophys. J. 665R, 607 (2004).

[51] U. Seljak, A. Slosar and P. McDonald, arXiv:astro-ph/0604335.

[52] J. Hamann et al., arXiv:0705.0440

[53] Jimenez, R., et al. 2004, MNRAS, 349, 240

[54] Jimenez et al., 2007, in preparation 